INTERNATIONAL JOURNAL OF SCIENTIFIC RESEARCH

COMPARATIVE ANALYSIS OF CYTOMORPHOLOGY PATTERNS AND AFB

POSITIVITY OF LYMPH NODE ASPIRATE VS CBNAAT FOR DETECTION OF TUBERCULAR LYMPHADENOPATHY"

\title{
Pathology
}

\section{Dr. Jayanti} Chandrakar

\section{Dr. Lokesh Sahu}

\section{Dr. Shraddha} Sahu*
Associate Professor, Department of Pathology, Pt. JNM Medical College and Dr. BRAM Hospital, Raipur (Chhattisgarh)

Postgraduate Student, Department of Pathology, Pt. JNM Medical College and Dr. BRAM Hospital, Raipur (Chhattisgarh)

Postgraduate Student, Department of Pathology, Pt. JNM Medical College and Dr. BRAM Hospital, Raipur (Chhattisgarh) *Corresponding Author

\section{ABSTRACT}

Background: TB remains a major health concern world wide with extrapulmonary TB accounting for $20 \%$ cases in India. Tubercular lymphadenopathy is most common extrapulmonary manifestation in TB. Aim: To compare and analyse cytomorphology patterns, AFB positivity and CBNAAT for detection of tubercular lymphadenopathy Methodology: a total of 80 FNAC samples from lymph nodes were taken, one part was sent for CBNAAT and other part was used to prepare slides and stained with H\& E, PAP, MGG, ZN stain for AFB. Results: Out of 80 cases, 52 $(65 \%)$ were granulomatous, $14(17.5 \%)$ each were of reactive and suppurative. Among these 52 cases reported as granulomatous 32 were positive for CBNAAT, $11 \mathrm{AFB}$ positive. Only 1 case out of 14 reactive cases was positive for CBNAAT. Out of 14 suppurative cases, 4 cases were CBNAAT positive. Conclusion: CBNAAT is a rapid test, can detect even if bacilli is less in number but FNAC along with AFB is simple procedure in resource limited area.

\section{KEYWORDS}

\section{CBNAAT, FNAC, AFB}

\section{INTRODUCTION}

Lymphadenopathy often occurs in the head and neck or inguinal areas and usually noted in clinical practice. Enlarged lymph nodes could be due to infection, metastatic malignancy or lymphoma. ${ }^{1}$ Tuberculous lymphadenitis is the most common Extrapulmonary (EP) manifestation of TB and the majority of cases have no active lung involvement. $^{2,3}$

Tuberculosis (TB) is an important health problem in low and middle income countries and remains a key challenge to public health and has an estimated global annual incidence of 9.6 million cases in India according to WHO annual report. ${ }^{4,5}$ Our ability to tackle this disease has been severely hampered by inadequate diagnostic assays ${ }^{4}$.No single diagnostic test is satisfying to all the demand of rapid, affordable and easy confirmatory test.

FNAC offers a feasible and safe option for specimen collection. Fine Needle Aspiration Cytology (FNAC) is an economical and reliable first line investigation in lymphadenopathy. FNAC is now considered as a valuable diagnostic aid as it gives early results, simple procedure, minimal trauma and complications. ${ }^{3}$

Culture can also be done but it is also time consuming.Histology is time-consuming and remains difficult to undertake and establish the diagnosis of TB with high specificity. ${ }^{6}$

The use of cytology together with the confirmation of acid fastness by Ziehl-Neelsen (ZN) staining and Papanicolaou stain-induced fluorescence microscopy as well as mycobacterial detection by culture offers excellent yields ${ }^{7,8}$ but remains limited by the absence of species confirmation, slow turnaround times, and/or lack of drug resistance guidance.

Several Nucleic Acid Amplification Technology (NAAT), tests have been developed that rapidly detect $\mathrm{M}$. tuberculosis DNA in patient samples and look for DNA changes that make M. tuberculosis drugresistant. ${ }^{9}$

Cartridge Based Nucleic Acid Amplification test (CB-NAAT), is an automated DNA test that detects $M$. tuberculosis and rifampicin resistance (an indicator of MDR-TB) within two hours for the investigation of patients who might have TB

In this study we compared the results of ZN stained smears obtained by FNAC of lymph nodes and CBNAAT for the diagnosis of tubercular lymphadenopathy.

\section{MATERIAL \& METHODS}

Study design- prospective, observational study

Duration of study- 1 year (September 2018-September 2019) Sample size- 80

Study area- patients with clinical suspicion of TB visiting OPD of various dept of Dr Bhim Rao Ambedkar hospital and Pt. J.N.M Medical college Raipur.

\section{Inclusion criteria:}

Enlarged and palpable lymph nodes with clinically presumptive TB sent for FNAC in dept of pathology, referred from any dept of Bhim Rao Ambedkar Memorial Hospital.

\section{Exclusion Criteria:}

Cases already taking treatment for TB, active or suspected malignancy, reactive lymphadenitis, terminal disease with poor prognosis, non- cooperative patients, patients not willing to give consent for study

The skin of the patient, over the suspected lymph node to be aspirated was cleaned and disinfected using spirit swabs. The lymph node was then fixed Following this, an 18 gauge needle attached to $10 \mathrm{ml}$ syringe was introduced into the lymph node and adequate material was aspirated. One part of this aspirated material was put in a sterile container and sent for CB-NAAT and the other part was smeared on slides. One smear slide was fixed in $95 \%$ ethyl alcohol for staining with Haematoxylin-Eosin and Papanicolaou stain, the other was air dried for Giemsa staining and few slides were kept aside for special stains i.e ZN stain for AFB for TB. The slides were examined under the microscope for visualization of granulomas and AFB.

\section{RESULT}

Table-1: Gross appearance in FNAC-

\begin{tabular}{|c|c|}
\hline Gross appearance & No. of cases \\
\hline Purulent & $30(37.5 \%)$ \\
\hline Bloody stained & $33(41.2 \%)$ \\
\hline Blood with pus & $17(21.3 \%)$ \\
\hline
\end{tabular}

Table 2: Results of Cytological pattern

\begin{tabular}{|c|c|c|}
\hline Cytology Pattern & Cases & Percentage \\
\hline Granulomatous & 52 & $65 \%$ \\
\hline Reactive & 14 & $17.5 \%$ \\
\hline Suppurative & 14 & $17.5 \%$ \\
\hline Total & 80 & $100 \%$ \\
\hline
\end{tabular}


Table 3: Subtype of Granulomatous lesion with AFB \& CBNAAT results

\begin{tabular}{|c|c|c|c|}
\hline Type & No. of cases & $\begin{array}{c}\text { AFB } \\
\text { Positive }\end{array}$ & $\begin{array}{c}\text { CBNAAT } \\
\text { Positive }\end{array}$ \\
\hline $\begin{array}{c}\text { Granuloma without } \\
\text { necrosis }\end{array}$ & $4(7.8 \%)$ & 00 & 0 \\
\hline Granuloma with necrosis & $33(63.4 \%)$ & $7(21.2 \%)$ & $19(57.5 \%)$ \\
\hline Only necrosis & $15(28.8 \%)$ & $4(26.6 \%)$ & $13(86.6 \%)$ \\
\hline Total & $52(100 \%)$ & 11 & 32 \\
\hline
\end{tabular}

Table 4: CBNAAT results

\begin{tabular}{|l|l|l|l|l|}
\hline CBNAAT & Positive & Percentage & Negative & Percentage \\
\hline Granulomatous (52) & 32 & $61.5 \%$ & 20 & $38.5 \%$ \\
\hline Reactive (14) & 1 & $7 \%$ & 13 & $93 \%$ \\
\hline Suppurative (14) & 4 & $28.5 \%$ & 10 & $71.5 \%$ \\
\hline Total & 37 & - & 43 & - \\
\hline
\end{tabular}

Present study included 80 subjects with cervical lymphadenopathy. FNAC done of that lymphnode was compared with ZN staining and CBNAAT findings. The age group ranges from 7-85 yrs with maximum number of cases between the age group of 21-40 years i.e 45 cases. Male female ratio was $1: 1.2$.

Gross appearance of aspirated material of FNAC is shown in table $1.41 .2 \%$ (33 cases) were bloody, purulent in $37.5 \%$ cases and $21.3 \%$ cases had a mixed aspirate of blood with pus.

The various cytological pattern seen in our study as shown in table 2 composed of predominantly granulomatous lymph node $(65 \%)$, reactive and suppurative LN being $17.5 \%$ each. In the present study we further sub-categorized granulomatous lymphadenopathy into 3 categories i.e. Granuloma with necrosis (33cases), Granuloma without necrosis (4 cases) and necrosis only (15 cases) as shown in table 3. $\mathrm{AFB}$ and CBNAAT was performed and it was negative in granulomatous without necrosis. AFB was positive in 7 cases while CBNAAT was positive in 19 cases of granuloma with necrosis. Smears that showed only necrosis had 4 cases AFB positive while 13 cases showed CBNAAT positive.

Overall CBNAAT results are shown in table 4. Granulomatous lesion showed $61.5 \%$ positive result, reactive lymph node $7 \%$ and suppurative lesion showed $28.5 \%$ positivity.

\section{DISCUSSION}

The annual global incidence of extra pulmonary TB has been increasing in the last decade due to changing TB control practices, spread of HIV and population growth. The diagnosis of EPTB poses particular challenge for clinicians because of atypical presentations and lacking health facilities.

The peak age group with granulomatous lymphadenitis was $21-40 \mathrm{yrs}$. The similar age distribution was found by Mulualem et al and Louis et $\mathrm{al}^{11,}$

Out of 80 cases $46.25 \%$ cases were positive for CBNAAT which was comparable with study of Gour et $\mathrm{al}^{12}$ and Srwar et al ${ }^{13}$ whereas higher percentage of CBNAAT positivity was found in studies of Moure et al , Gupta et $\mathrm{al}^{15}$ and Ligthelm et $\mathrm{al}^{16}$

Of the 80 patients included in this study, cytomorphological features associated with TB were 52 patients with majority being females. Out of 52 patients $32(61.5 \%)$ showed CBNAAT positivity while ZN was positive in $11(21.1 \%)$ cases.

Out of the 14 reactive lesions and 14 suppurative lesion in FNAC; CBNAAT was positive in $1(7 \%)$ and $4(28.5 \%)$ cases respectively. Thus the lesions that were not identified as of tubercular lesion in FNAC were detected by CBNAAT, thereby showing the superiority of CBNAAT over FNAC.

CBNAAT was able to detect $\mathrm{TB}$ in $6.25 \%$ ( 1 reactive +4 suppurative $=5 / 80$ ) of cases which were not diagnosed by FNA, similarly Sunil et al studied 289 cases out of which 19 cases were false negative in FNAC, thus CBNAAT detected $6.5 \%$ of cases which were negative in FNAC

$\mathrm{ZN}$ stain for AFB was positive in $21.1 \%$ cases of the granulomatous lymphaedititis. Overall out of the 80 cases $18 \%$ cases were $\mathrm{ZN}$ positive which was comparable with the study of Subhan Ali et al, Brijesh thakur et al and anshu et $\mathrm{ll}^{17}$ while in study of Majid momin et al it was positive in $62 \%$ of cases

FNAC is a simple procedure which can be performed in an outpatient setting and is ideal in resource limited settings ${ }^{18}$. Specimen collection is simple, economical, easy to do and safe procedure ${ }^{19}$. But in a tertiary level hospital we cannot depend solely on FNAC for the diagnosis of tuberculosis.

During the last decade, a number of Nucleic acid amplification (NAA) methods have been developed for rapid detection and identification of MTB ${ }^{20}$. newly administered Gene-xpert MTB/RIF assay take two hours to provide the final results, in addition distinguishes MTB and mycobacteria other than tuberculosis and also provide rifampicin resistance simultaneously ${ }^{10}$ Polymerase chain reaction (PCR) is based on NAA methods and is widely used for rapid diagnosis of TB..$^{21}$ This study supports the routine use of CBNAAT for the diagnosis of Extra pulmonary tuberculosis as time factor and drug resistance are the two important factors for the diagnosis and treatment of tuberculosis.

Though CBNAAT has several disadvantages there are few limitations like high cost, inability to differentiate MDR-TB and XDR-TB, not suitable for monitoring response to treatment and suggestive of conventional microscopy and culture are required for monitoring MDR-TB and few technical problems like requirement of stable electricity supply, limited temperature range and maintenance.

Culture being gold standard but takes 4-6 weeks for diagnosis. Newer faster radiometric (BACTEC) and Non- radiometric methods like fluorescent labelled mycobacterium growth indicator test (MGIT) methods being used and provide results in 7-10 days. It is time consuming and equipment expenses are much high, more skilled personals, continuous monitoring for several days and further confirmation of positive cultures by making $\mathrm{ZN}$ smears are required. ${ }^{22}$

\section{CONCLUSION}

To conclude a high clinical suspicion and special diagnostic procedures are required for diagnosis of extrapulmonary tuberculosis. FNAC and ZN microscopy is the first line investigation and is also simple and safe procedure especially in resource limited area. Culture is gold standard but is time consuming. CBNAAT being the latest technique is less time consuming, can detect TB even if bacilli are less in number and also provides information about rifampicin resistance. But CBNAAT has a disadvantage of being costly, maintenance is high. To conclude combined techniques proved itself as an effective tool in OPD basis diagnosis and early initiation of treatment to prevent morbidity, mortality and economic loss.

\section{REFERENCES}

1. MukulPatar et al(2014). A clinicopathological evaluation of cervical lymphadenopathy in children (0-14 Years) By fine needle aspiration cytology and histopathological examination - A hospital based study. National Journal of Otorhinolaryngology and Head \& Neck Surgery.2014;2(11):12-14.

2. Ghartimagar D et al(2011). Utility of fine needle aspiration cytology in metastatic lymph nodes. Journal of Pathology of Nepal.2011;1(2):92-95

3. Louis J.LigthelmXpert MTB/RIF for Rapid Diagnosis of Tuberculous Lymphadenitis from Fine-Needle-Aspiration Biopsy Specimens. Journal of clinical microbiology, Nov. 2011,p. 3967-3970 Vol. 49, No. 110095-1137. doi:10.1128/JCM.01310-11.

4. Ardizzoni $\mathrm{E}$ et al(2015). Implementing the Xpert $(\mathrm{R})$ MTB/RIF Diagnostic Test for Tuberculosis and Rif . Tuberculosis and Rifnes pountries. 0144656 . eCollection 2015. [PubMed] pone.0144656. eCollection 2015. [PubMed]

. Catherina CB, Rapid molecular detection of TB and Rifampicin resistance, NEJM, 2010, Sep; 363:11. Doi: 10.1056/NEJMoa0907847.

6. Lee JY.(2015) Diagnosis and treatment of extrapulmonary tuberculosis. Tuberc Respi Dis (Seoul). 2015 Apr;78(2):47-55. doi: 10.4046/trd.2015.78.2.47. Epub 2015 Apr 2 [PubMed]

7. Wright C. A., et al. (2008). Diagnosing mycobacterial lymphadenitis in children using fine-needle aspiration biopsy: cytomorphology, ZN staining and autofluorescence - making more of less. Diagn. Cytopathol, 36:245-251 [PubMed] [Google Scholar]

8. Wright C. A et al,(2004. Mycobacterial autofluorescence in Papanicolaou-stained lymph node aspirates: a glimmer in the dark? Diagn. Cytopathol. 30:257-260 [PubMed] [Google Schola

9. Singh KG et al (2017) Role of CB-NAAT in diagnosing Mycobacterial tuberculosis and rifampicin resistance in tubercular peripheral lymphadenopathy. Int J Med Res Rev 2017;5(03):242-246.doi:10.17511/ijmrr. 2017.i03.05.

10. majid momin et al (2017) comparitive analysis of gene expert assay in addition to ZN microscopy for detection of extrapulmonary TB in fine needle aspiration samples. Sch.J.App.Med.Sci.,Jul2017;5(7D):2803-2808.

11. Mulualem et al. Genexpert MTB/RIF Assay for the diagnosis of tuberculous lymphadenitis on concentrated fine needle aspirates in high tuberculosis burden settings

12. Gour sanjay et al(2017). Genotypic diagnosis of extrapulmonary tuberculosisCBNAAT a novel tool. Medpulse Int J Med 2017;4(2):79-82

13. Srwar A et al (2014)Rapid detection of Mycobacterium tuberculosis and rifampicin resistance in extrapulmonary samples using Genexpert MTB/RIF assay. IOSR. J Denta 
Med Sci 2014;13(11):50-53.

14. Moure R et al(2012). Effectiveness of an integrated real time PCR method for detection of mycobacterium tuberculosis complex in smear negative extrapulmonary samples in an area of low tuberculosis prevalence. J Clin microbiol 2012:50(2):513-515

15. Gupta $\mathrm{S}$ et al(2018). Role of gene Xpert Mtb/Rif in diagnosis of tuberculosis pus. Int J Curr res Med Sci 2018;4(2):81-85

16. Ligthelm L et al (2011). Xpert MTB/RIF for the rapid diagnosis of tuberculous lymphadenitis from fine needle aspiration biopsy specimens. J Clin Microbiol 2011;49(11):3967-3970.

17. Kumar anshu et al (2018). A study on role of cartridge based nucleic acid amplification test (CBNAAT) for diagnosing pediatric tuberculosis in a tertiary care hospital in eastern India.J of pediatrics \& neonatology ISSN 2474-7521

18. Wright $C$ et al (2009). Fine needle aspiration biopsy: an undervalued diagnostic modality in paediatric mycobacterial disease. Int. J. Tuberc. Lung Dis. 13:1467-1475

19. Gupta PP et al. (2017)Efficacy of cartridge based nucleic acid amplification test to diagnose tubercular pleural effusion. Int J Res Med Sci. 2017 Aug;5(8):3637-3643

20. Piersimoni $\mathrm{C}$ et al (2002).performance assessment of two commercial amplification assays for direct detection of mycobacterium tuberculosis complex from respiratory and extrapulmonary specimens. J. OfClin microbiol.2002 Nov 1;40(11):4138-42

21. automated real time nucleic acid amplification technology for rapid and simultaneous detection of tuberculosis and rifampicin resistance : xpert MTB/RIF system: policy statement; WHO 2011

22. Theron $G$ et al (2012). Do adjunct tuberculosis tests, when combined with xpert MTB/RIF, improve accuracy and cost of diagnosis in a resourse poor setting?. European respiratory journal. $2012 \mathrm{Jul} 1 ; 40(1): 161-8$. 